\title{
P.09 - DIFFERENCES IN INSIGHT LEVELS AMONG PATIENTS HOSPITALIZED WITH PSYCHOSIS IN ACUTE CARE
}

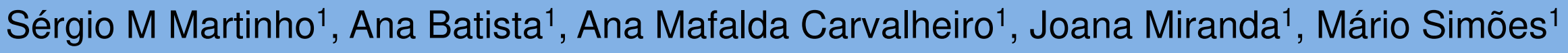 \\ Psychiatry and Mental Health Department, Leiria Hospital Centre, Leiria, Portugal
}

\section{Introduction}

Insight is defined as the patient's capacity to understand the nature, significance and severity of his or her illness ${ }^{1}$. Impaired insight is a common feature among individuals suffering from psychosis, particularly in schizophrenia ${ }^{2}$. Higher insight has been associated with older age, female gender and white ethnicity, while lesser insight is found in patients with more severe psychopathology ${ }^{3}$. Besides this, impaired insight plays an important role in reduced treatment adherence and poorer outcomes ${ }^{4,5}$. With this study, we aim to find associations between insight levels and admission related variables in a sample of patients with psychotic features.

\section{Methods}

In a convenience sample, we invited 39 patients hospitalized with psychotic features to complete the Marková and Berrios Insight Scale 6 . This scale measures the insight level, varying from 0 to 30 points; higher scores mean higher levels of insight. Assistant clinicians were blind for the results. As insight levels presented with a normal distribution, we utilized Student's t-test to ascertain differences in means between groups. Correlations were established through Spearman's coefficient. Statistical significance was set at $p<0.05$.

\section{Results}

\begin{tabular}{|c|c|}
\hline Variable & $n(\%)$; Mean (SD)*; Median (IQR) ${ }^{\star \star}$ \\
\hline Age (years) ${ }^{* *}$ & $37(21)$ \\
\hline Insight Level (Units)* & $12.9(5.6)$ \\
\hline Length of Stay (Days)* & $22.3(8.7)$ \\
\hline № of Total Oral Drugs/Day at Discharge & $3(3)$ \\
\hline \multicolumn{2}{|l|}{ Gender } \\
\hline Male & $26(66.7)$ \\
\hline Female & $13(33.3)$ \\
\hline \multicolumn{2}{|l|}{ Compulsory Admission } \\
\hline Yes & $18(46.2)$ \\
\hline No & $21(53.8)$ \\
\hline \multicolumn{2}{|l|}{ Admission Following Police Referral } \\
\hline Yes & $14(35.9)$ \\
\hline No & $24(61.5)$ \\
\hline \multicolumn{2}{|l|}{ Family History of Mental IIIness Reported } \\
\hline Yes & $15(38.5)$ \\
\hline No & $24(61.5)$ \\
\hline \multicolumn{2}{|l|}{ Living Alone } \\
\hline Yes & $9(23.1)$ \\
\hline No & $30(76.9)$ \\
\hline \multicolumn{2}{|l|}{ ICD10 Diagnostic Group } \\
\hline F10-F19 & $2(5.1)$ \\
\hline F20-F29 & $29(74.4)$ \\
\hline F30-F39 & $8(20.5)$ \\
\hline \multicolumn{2}{|l|}{ Exiting with Long Acting Injectable } \\
\hline Yes & $28(71.8)$ \\
\hline No & $11(28.2)$ \\
\hline
\end{tabular}

\section{Results}

\begin{tabular}{lcc}
\hline Variable & $\begin{array}{c}\text { Insight Level } \\
\text { (mean) }\end{array}$ & $\boldsymbol{p}$ \\
\hline $\begin{array}{l}\text { Compulsory Admission } \\
\text { Yes }\end{array}$ & 10.1 &. $\mathbf{0 2}$ \\
$\quad$ No & 14.3 & \\
\hline Admission Following Police Referral & & .047 \\
$\quad$ Yes & 9.9 & \\
$\quad$ No & 13.7 & \\
\hline $\begin{array}{l}\text { Family History of Mental Illness Reported } \\
\text { Yes }\end{array}$ & 14.8 & \\
$\quad$ No & 10.9 & \\
\hline Living Alone & & .15 \\
$\quad$ Yes & 10.0 & \\
$\quad$ No & 13.1 & \\
\hline Exiting with Long Acting Injectable & & .52 \\
Yes & 12.8 & \\
$\quad$ No & 11.5 & \\
\hline
\end{tabular}

There was a positive correlation between the total number of oral psychotropic drugs/day at discharge and the level of insight $(\rho=.345 ; p=.03)$.

\section{Conclusions}

Insight remains as a complex concept to assess, due to its multidimensionality. Our results demonstrate that patients' insight may play a role in the decision to activate compulsory admission. We also note that clinicians may feel more confident about prescribing more complex treatment plans at discharge in individuals with higher levels of insight.

\section{References:}

1.Jacob K. Insight in psychosis: An indicator of severity of psychosis, an explanatory model of illiness, and a coping strategy. Indian J Psychol Med. 2016

2. David AS. Insight and psychosis. Br J Psychiatry. 1990;156(6):798-808.

3. McEvoy JP, Johnson J, Perkins D, Lieberman JA, Hamer RM, Keefe RSE, et al. Insight in first-episode psychosis. Psychol Med. 2006;36(10):1385-93. 4. Gharabawi GM, Lasser RA, Bossie CA, Zhu Y, Amador X. Insight and its relationship to clinical outcomes in patients with schizophrenia or
schizoaffective disorder receiving long-acting risperidone. Int Clin Psychopharmacol. 2006;21(4). 5. Byrne MK, Deane FP. Enhancing patient acherence: Outcomes of medication alliance training on therapeutic alliance, insight, adherence, and
psychopathology with mental health patients. Int $J$ Ment Health Nurs. 2011:20(4):284-95. 6. Vanelli I, Chendo I, Levy P, Figueira ML, Góis C, Santos J, et al. Adaptação Ipara português da escala de insight marková e berrios. Acta Med Port.
2010;23(6):1011-6. 